\title{
Modeling of Optoacoustic Signal Generation for High Resolution Near-Surface Imaging with Experimental Verification
}

\author{
Gopi Maguluri, Lei Sui, Ronald A. Roy, and Todd W. Murray* \\ Center for Subsurface Sensing and Imaging Systems \\ Department of Aerospace and Mechanical Engineering, Boston University, \\ 110 Cummington Street, Boston, MA, 02215, USA
}

\begin{abstract}
Optoacoustic systems making use of optical detection probes are potentially advantageous over contact transducers for noncontact, noninvasive high-resolution near surface imaging applications. In this work, an interferometer is used for high-frequency optoacoustic microscopy. The limitations of this system in terms of both sensitivity and resolution are discussed. A theoretical model has been developed for two-dimensional excitation source geometries, which can be used to predict the optoacoustic signal from a target material with an arbitrary through-thickness optical absorption distribution. The model incorporates the temporal and spatial profile of the excitation laser pulse, and is used to predict the actual out-of-plane displacement at the target surface. An adaptive, photorefractive crystal-based interferometry system has been used to measure the optically induced displacement on the surface of target materials, and the results show reasonable quantitative agreement with theory. The detection system has a $200 \mathrm{MHz}$ bandwidth allowing for highresolution imaging, and the use of optical probes for both generation and detection allows for the probes to be easily coaligned on the sample surface. Preliminary experimental results are presented demonstrating the feasibility of using alloptical optoacoustic microscopy for near surface imaging of small-scale spatial variations in optical absorption.
\end{abstract}

Keywords: Optoacoustic imaging, microscopy, photorefractive crystal, optical probe

\section{INTRODUCTION}

Optoacoustic imaging is a relatively new noninvasive imaging modality that combines many of the advantages of both optical and ultrasonic imaging techniques. A laser source is used to illuminate a tissue volume, and the absorption of light with-in the media leads to heating, thermal expansion, and acoustic wave generation. The acoustic wave field is measured on the surface of the material, and these signals are then related to the subsurface absorption distribution. The technique allows for the measurement of subsurface optical properties, thus taking advantage of the large optical contrast observed between tissue types. It also opens up the possibility of functional imaging, such as the measurements of tissue oxygenation or blood hemoglobin concentration. The acoustic wave field is much less sensitive to scattering than the optical field, and serves to transport the local absorption information to the detection region. Contact transducers are the predominant means of detecting optoacoustic transients. The transducer is coupled to the tissue surface through an appropriate coupling media, and used to measure the transient pressure at the surface. Assuming conditions of thermal and stress confinement, the spatial resolution that can be achieved in optoacoustic imaging is limited by the bandwidth of the detection system and the spatial extent of the detection probe. In order to resolve subsurface optical inhomogeneities on the order of $3 \mu \mathrm{m}$, for example, a detection bandwidth on the order of $250 \mathrm{MHz}$ is required. Several wideband optoacoustic contact transducers have been developed in recent years [1-3]. However, detection of optoacoustic transients with both axial and lateral resolutions in the $10 \mu \mathrm{m}$ range or less remains a formidable task, particularly in a backward mode detection configuration where the source and detector are co-located on the target surface. Optical detection of acoustic transients provides one potential means of overcoming this

*Prof. Todd W. Murray, Email: twmurray@bu.edu; Phone: 617-353-3951 
limitation. Optical detection offers a truly remote and noncontact alternative to contact transducers, and optical detection systems can be designed with bandwidths exceeding several hundred megahertz, thus effectively covering the useful frequency range for medical imaging applications. In addition, the detection probe can be tightly focused on the sample surface serving as a point detector. Array detection systems have also been demonstrated which allow for simultaneous detection of displacement at multiple points on a sample surface, and three dimensional optical detection arrays with sub-micrometer element size and micrometer scale element spacing may potentially be developed. Finally, optical detection probes and excitation sources can be co-located on the sample surface with relative ease, thus facilitating backward mode detection. While optical detection schemes are promising in terms of resolution, further developments are required in order to overcome the sensitivity limitations of optical detection systems before they are suitable for a wide range of optoacoustic imaging applications.

Several researchers have explored optical detection techniques [4-9]. Hamilton et al. have proposed two alternatives for the detection of high frequency ultrasound: active interferometric detection across the surface of a pellicle [4] and an etalon based detection scheme [5]. Paltauf et al. [6] used an optical reflectance method based on acoustically induced change in refractive index at the interface of glass and water demonstrating a $10 \mu \mathrm{m}$ spatial resolution. Recently Beard et al. [7] have developed a polymer film based Fabry-Perot detection system demonstrating a frequency range up to 20 $\mathrm{MHz}$ along with an angle scan technique that replaces the difficulty of mechanical scanning [8]. In the techniques mentioned above, the optical probe is coupled to the specimen through a sensor head or film. Measurements of optoacoustic signals in a truly remote manner, through collection of light scattered directly off of the sample surface, have been limited. This is in part due to the fact that in tissue or tissue-like media, the low amount of light that can be collected from the surface places rather severe limitations on the technique. However, remote detection of optoacoustic transients has been demonstrated by several groups [9-11]. Payne et al. [9] used a modified Mach-Zender interferometer for the detection of subsurface optical inhomogeneities in tissue phantoms, and the authors have developed an optoacoustic detection system using a photorefractive crystal based interferometer for the detection of out-of-plane displacements created by the ultrasonic transients [11].

In this work, we evaluate the feasibility of near-surface, all-optical optoacoustic microscopy. The optical excitation source is an Nd:YAG microchip laser with a 1.6ns pulse width, suitable for generating acoustic transients in the hundreds of $\mathrm{MHz}$ range. The pulse energy of this laser is $3 \mu \mathrm{J}$, several orders of magnitude lower than typically used for optoacoustic imaging applications. The optical detection system uses a photorefractive crystal based interferometer and was operated at a bandwidth of $200 \mathrm{MHz}$. The noncontact, broadband nature of the system make it well suited for nearsurface imaging applications including, for example, dermatological applications, skin cancer depth profiling, and burn characterization. In addition, the surface displacements produced by the microchip laser are evaluated using a model for pulsed optoacoustic generation in media with through thickness variations in optical absorption, and the results compared to experiments for layered polymer samples.

\section{THEORY}

The production of optoacoustic transients using a pulsed laser source is well understood and has been described in detail in the literature [12-16]. In this work, the integral transform technique has been adopted to solve for the transient normal displacement of the sample surface (the displacement component measured experimentally using an interferometer) produced by a cylindrically symmetric excitation source. Optoacoustic signal generation is described by the following equations:

$$
\begin{gathered}
u=\nabla \varphi, \\
\nabla^{2} \varphi-\frac{1}{c^{2}} \frac{\partial^{2} \varphi}{\partial t^{2}}=\gamma T, \\
\frac{\partial T}{\partial t}=\frac{q}{\rho C_{p}},
\end{gathered}
$$


where $\nabla^{2}=\frac{\partial^{2}}{\partial z^{2}}+\frac{\partial^{2}}{\partial r^{2}}+\frac{1}{r} \frac{\partial}{\partial r}, u$ is the displacement vector, $\varphi$ is the displacement potential, $T$ is the temperature, $\rho$ and $C_{p}$ are the density and specific heat at constant pressure for the medium, $c$ is the acoustic wave velocity and $\gamma$ is the volume coefficient of thermal expansion of the material. The two dimensional problem is formulated in cylindrical coordinates with $r$ in the radial direction and $z$ into the medium. Here we have assumed that thermal diffusion is negligible on the time scale of interest such that the temperature distribution directly follows the absorption distribution (thermal confinement) [17]. Considering a medium of finite thickness $h$, traction free boundary conditions are applied at the top and bottom surfaces:

$$
\rho \frac{\partial^{2} \varphi}{\partial t^{2}}=\left.0\right|_{z=0, z=h}
$$

The source function used to represent the incident laser pulse is given by [15]:

$$
q=E\left(1-R_{i}\right)\left[(A(z))\left(\frac{2}{\pi R^{2}} e^{-\frac{2 r^{2}}{R^{2}}}\right)\left(\frac{8 t^{3}}{\tau^{4}} e^{-\frac{2 t^{2}}{\tau^{2}}}\right)\right],
$$

where $E$ is the laser pulse energy, $R_{i}$ is the surface reflectivity of the material, $R$ is the Gaussian beam radius (radius of the pulse at $1 / \mathrm{e}^{2}$ of peak intensity), $\tau$ is the pulse width, and $\chi$ is the absorption coefficient of the material. In Eq. 5 the term $E\left(1-R_{i}\right)$ represents the absorbed energy in the medium. The first of the three terms in the square bracket describes the absorption distribution of light in the medium. For a single homogeneously absorbing layer buried at a depth $\mathrm{d}_{1}$ and extending to $d_{2}$, the absorption distribution can be written as the difference between two Heaviside step functions as follows:

$$
A(z)=\chi\left[H\left(z-d_{1}\right)-H\left(z-d_{2}\right)\right] e^{-\chi\left(z-d_{1}\right)}
$$

The axial displacement at the surface of the medium can be obtained by:

$$
u_{z}=\frac{\partial \varphi}{\partial z}
$$

Taking the Laplace transform with respect to time and Hankel transform of zero order with respect to space in the radial direction, the axial displacement for a layer described in Eq. 6 can be expressed as:

$\left.u_{z}^{L, H}\right|_{z=0}=\frac{e^{\chi d_{1}} \gamma \chi q^{L, H}}{2 \rho C_{p} s\left(\alpha^{2}-\chi^{2}\right) \sinh (\alpha h)}\left[(\alpha-\chi) e^{\alpha h}\left\{e^{-d_{2}(\alpha+\chi)}-e^{-d_{1}(\alpha+\chi)}\right\}+(\alpha+\chi) e^{-\alpha h}\left\{e^{d_{2}(\alpha-\chi)}-e^{d_{1}(\alpha-\chi)}\right\}\right]$,

where $s$ is the Laplace transform variable in the pair $(t, s)$ and $p$ is the zero order Hankel transform variable in the pair $(r, p)$, The superscripts $L$ and $H$ represent the parameter to be in Laplace and Hankel transform domains, Here $\alpha^{2}=p^{2}+\frac{s^{2}}{c^{2}}$

Eq. 8 can be simplified by taking the slab to be semi infinite $(h \rightarrow \infty)$ and is given by:

$$
\left.u^{L, H}\right|_{z=0}=\frac{e^{\chi d_{1}} \gamma \chi q^{L, H}}{\rho C_{p} s(\alpha+\chi)}\left[e^{-d_{2}(\alpha+\chi)}-e^{-d_{1}(\alpha+\chi)}\right]
$$

It is straightforward to extend this analysis to the case of a multilayered media by constructing a stack of homogenously absorbing layers [18]. The absorption distribution of an $n$ layered system can be described as: 


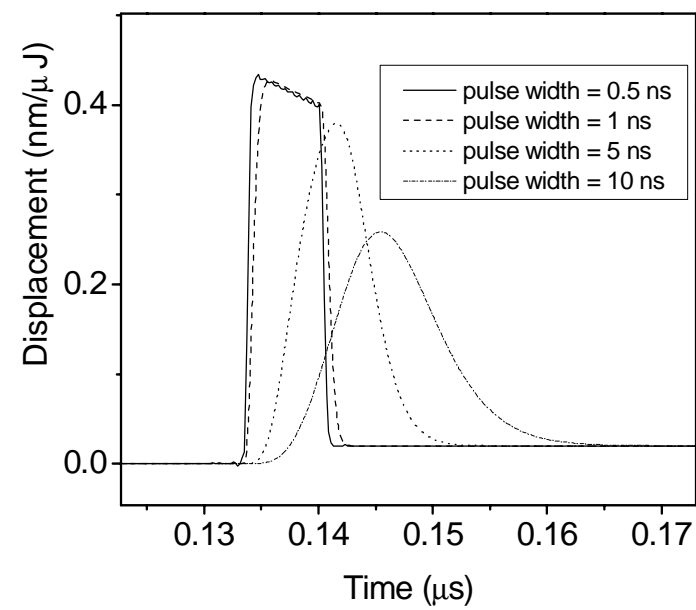

Figure 1. Effect of the pulse width on the ultrasonic displacement at the surface for cylindrically symmetric particle (dia. $10 \mu \mathrm{m}$ and thickness $10 \mu \mathrm{m}$, absorption coefficient $10 \mathrm{~mm}^{-1}$ ) buried at a depth of $200 \mu \mathrm{m}$

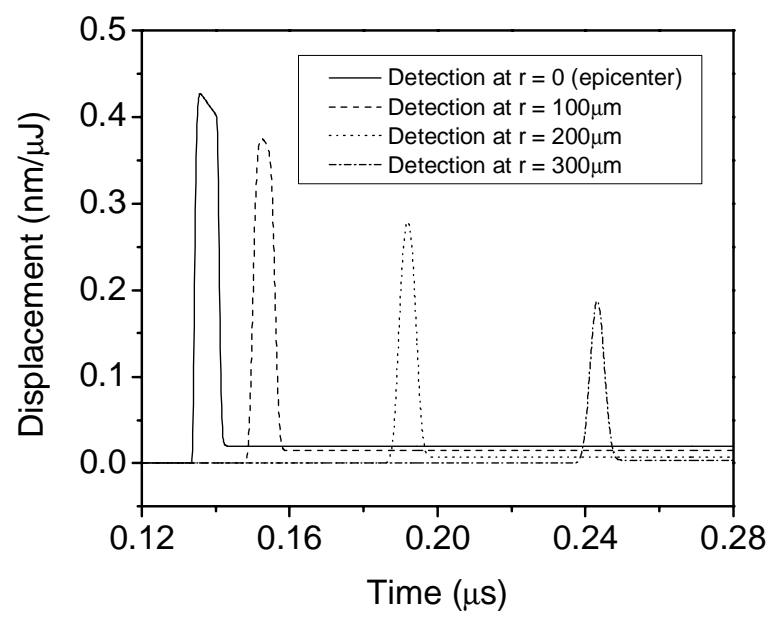

Figure 2. Off epicenter surface displacements, moving from the epicenter in steps of $100 \mu \mathrm{m}$ for the same particle as described in Figure 1 with a pulse width of $1 \mathrm{~ns}$

$$
\begin{aligned}
& A(z)=\chi_{1}\left[H(z)-H\left(z-d_{1}\right)\right] e^{-\chi_{1}(z)}+e^{-\chi_{1} d_{1}} \chi_{2}\left[H\left(z-d_{1}\right)-H\left(z-d_{2}\right)\right] e^{-\chi_{2}\left(z-d_{1}\right)}+\ldots \\
& \chi_{n} e^{-\chi_{1} d_{1}-\chi_{2}\left(d_{2}-d_{1}\right) \ldots-\chi_{n-1}\left(d_{n-1}-d_{n-2}\right)}\left[H\left(z-d_{n-1}\right)-H\left(z-d_{n}\right)\right] e^{-\chi_{n}\left(z-d_{n-1}\right)}
\end{aligned}
$$

where $\chi_{n}$ is the absorption coefficient of $\mathrm{n}^{\text {th }}$ layer. The transient, time domain displacement is found through numerical inversion of the transform domain solution.

Theoretical results are shown below for a variety of cases using the model. For all the cases, the nominal values of the parameters (unless otherwise specified) match that of gel phantoms and are taken as: longitudinal wave velocity $c=$ $1.5 \mathrm{~mm} / \mu \mathrm{s}$, density $\rho=1.045 \mathrm{~g} / \mathrm{cm}^{3}$, specific heat at constant pressure $C_{p}=3.71 \mathrm{~J} / \mathrm{gK}$, volume coefficient of thermal expansion $\gamma=2.07 \times 10^{-4} \mathrm{~K}^{-1}$, pulse width of $\tau=1 \mathrm{~ns}$, and distance off of epicenter $\mathrm{r}=0$. Figure 1 illustrates the effect of laser pulse width on the optoacoustic signal generated by a small absorber buried $200 \mu \mathrm{m}$ below the surface. To simulate the absorber, the laser spot size is reduced to a radius of $5 \mu \mathrm{m}$, and the absorption takes place in a $10 \mu \mathrm{m}$ thick absorbing laser with an absorption coefficient of $10 \mathrm{~mm}^{-1}$. The surface displacement calculated for several pulse widths is shown in Figure 1. For a sufficiently short pulse width, the displacement in this case follows the absorption distribution in the media, as expected. As the pulse length is increased, such that it is long with respect to the acoustic transient time in the absorption region, the displacement follows the incident laser pulse profile. The displacement is given in units of nm/ $\mu \mathrm{J}$ of incident laser energy and the displacements are quite large due to the fact that the source is tightly focused. Note that the shift in the arrival time is an artifact of the model, where zero time is defined with respect to the start of the excitation pulse rather than the peak. Figure 2 illustrates the change in the predicted displacement as the receiver is moved off of epicenter. The smoothing of the signals predicted off of epicenter is primarily due to the fact that the source region has well defined boundaries in the axial direction, but has a Gaussian transverse profile. An image of the absorption region can be obtained through back-propagation using, for example, a simple delay and sum algorithm.

The displacement signal produced by a cylindrically symmetric disk (dia. $1 \mathrm{~mm}$ and thickness $25 \mu \mathrm{m}$ ) of absorption coefficient $2.3 \mathrm{~mm}^{-1}$ buried inside of a media with background absorption of $2 \mathrm{~mm}^{-1}$ is shown in Figure 3 . Three signals

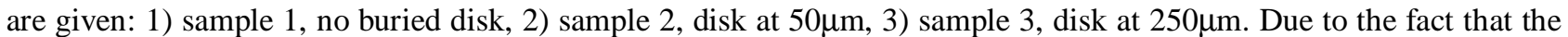
laser source is relatively large, the absorption distribution is approximately 1-D and the displacement signal, in this case, does not follow the absorbed energy distribution but rather the integral of this distribution. The signal is dominated by the background absorption in the media, and the effect of the absorbing layer can be observed in the zoomed in plots 

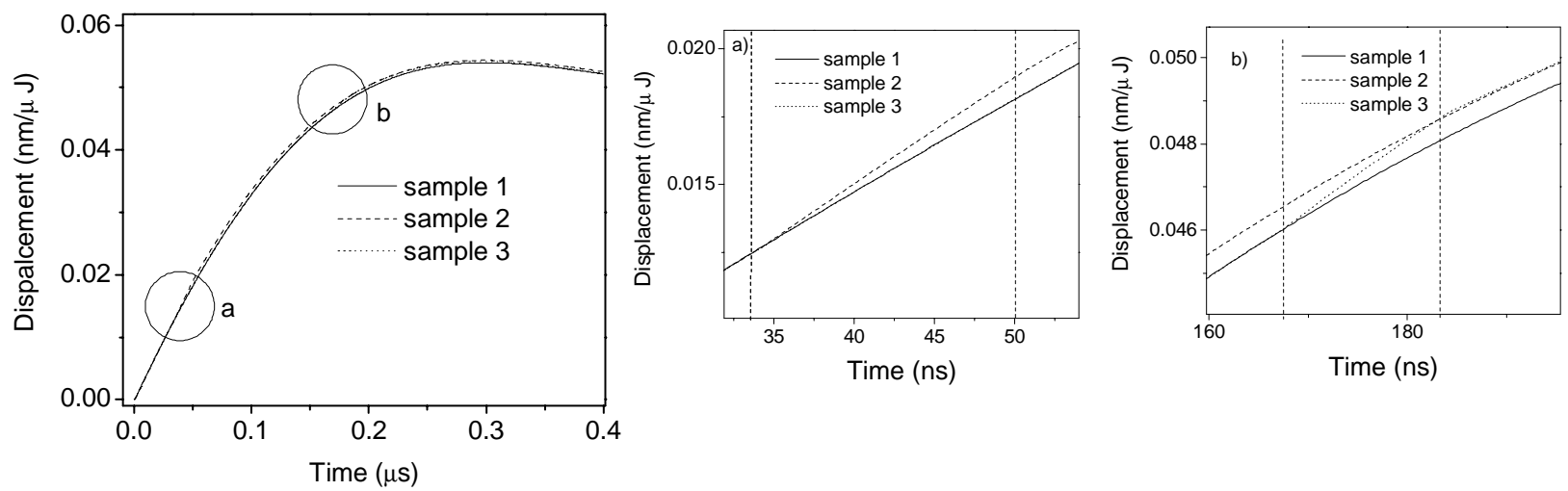

Figure 3. Effects on surface displacement due to absorbing layers buried at different depths in the medium. Absorbing layer is a cylindrically symmetric disk (dia. $1 \mathrm{~mm}$ and thickness $25 \mu \mathrm{m}, \chi=2.3 \mathrm{~mm}^{-1}$ ). Sample 1: Homogeneously absorbing medium with $\chi=2 \mathrm{~mm}^{-1}$. Sample 2: Absorbing layer buried at a depth of $50 \mu \mathrm{m}$ from the surface in sample 1. Sample 3: Absorbing layer buried at a depth of $250 \mu \mathrm{m}$ from the surface in sample 1.

shown in Figures 3a,b. The vertical lines on the plots indicated the arrival time from the front and back surface of the buried absorber. In Figure 3a, at the arrival time of the acoustic wave from the front surface of the absorber, the displacement observed in sample 2 begins to increase. Between the arrival from the front and back surface, the signal continues to increase over the background, as it follows the integral of the absorption profile. Following the arrival from the back surface, the signal returns parallel to that in the homogeneous absorber, but offset by approximately $5 \%$. The signals predicted from a buried absorber with the same dimensions as in Figure 3, buried at a depth of $100 \mu \mathrm{m}$, are shown in Figure $4 \mathrm{a}$ as a function of absorption coefficient in the disk. The absorption coefficient in the background material is $2 \mathrm{~mm}^{-1}$. One observes a maximum displacement difference of approximately $3 \%$ between the baseline background signal, and the signal with the absorber, when the absorption coefficient of the absorber is $2.3 \mathrm{~mm}^{-1}$. The ability to detect such a signal depends on both the amount of energy in the excitation source, and the amount of light collected from the sample surface. Figure $4 \mathrm{~b}$ shows the derivative of the signal from the buried absorber $\left(2.3 \mathrm{~mm}^{-1}\right)$. The derivative clearly follows the absorption profile in this case.
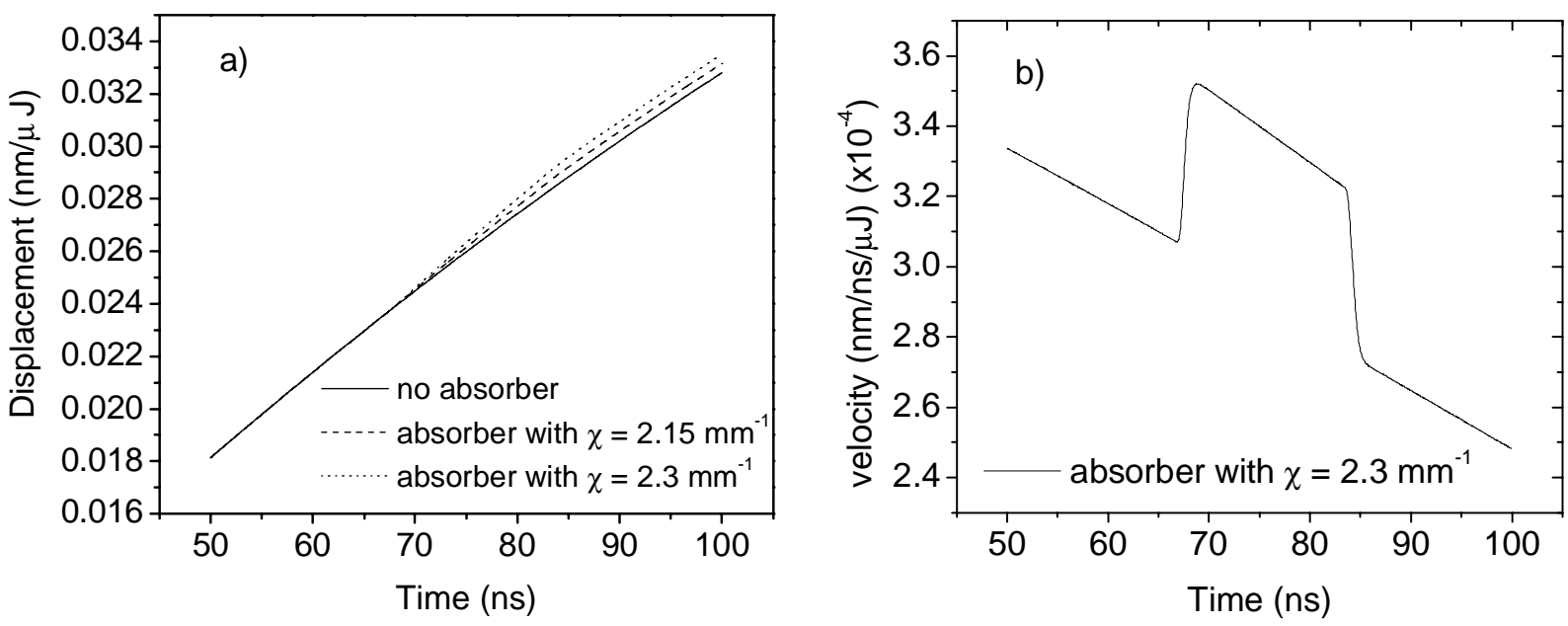

Figure 4. Effect of optical contrast a) Variation in surface displacement due to absorbing layers with different optical contrast (with absorption coefficient $2.15 \mathrm{~mm}^{-1}$ and $2.3 \mathrm{~mm}^{-1}$ ) in the background medium (with absorption coefficient $2 \mathrm{~mm}^{-1}$ ). Absorbing layer: cylindrically symmetric disk (dia. $1 \mathrm{~mm}$ and thickness $25 \mu \mathrm{m}$, located at $100 \mu \mathrm{m})$. b) Velocity for the sample with absorber having absorption coefficient $2.3 \mathrm{~mm}^{-1}$. 


\section{EXPERIMENTAL SETUP}

A schematic of the optoacoustic microscopy system is shown in Figure 5. The laser excitation source is an Nd:YAG microchip laser with energy per pulse of $3 \mu \mathrm{J}$ and a pulse repetition frequency of approximately $3 \mathrm{kHz}$. The laser source is weakly focused and sent through a dichroic mirror to the sample surface. The dichroic mirror transmits light at 1064 $\mathrm{nm}$ and reflects light at $532 \mathrm{~nm}$. A continuous wave (CW) laser (Light Wave, 2001, 200mW) operating at 532nm wavelength is used for the detection of the ultrasonic transients on the sample. A polarizing beam splitter (PBS) and a halfwave plate (HWP) are used to split the light into signal and reference beams. The signal beam is focused on to the sample using an objective ( $5 \mathrm{X}, \mathrm{NA}=0.14)$ and the reflected light, which is phase modulated by the acoustically induced surface displacement, is redirected, using polarized beam splitter and a quarter wave plate (QWP), into the photorefractive crystal (PRC). The photorefractive crystal used in this system is Bismuth Silicon Oxide (BSO) with dimensions of $5 \mathrm{~mm} * 5 \mathrm{~mm} * 7 \mathrm{~mm}$. The crystal is cut in an orientation such that the space charge field is aligned along the $<001>$ axis of the crystal; the crystallographic configuration is often referred to as a holographic cut. A high voltage DC field $(4 \mathrm{KV}-\mathrm{cm})$ is applied to the crystal to the crystal through a high voltage amplifier (TREK, 609E-6). The signal and reference beams interfere at the photorefractive crystal and an index grating is written through the photorefractive effect. The reference beam diffracts from this grating in the two-wave mixing process. The diffracted reference is wavefront matched to the transmitted signal and the two beams interfere at the photodetector. Under the application of a DC field, the diffracted reference beam is phase shifted by 90 degrees with respect to the transmitted signal beam, allowing for high sensitivity detection of signal beam phase modulation.

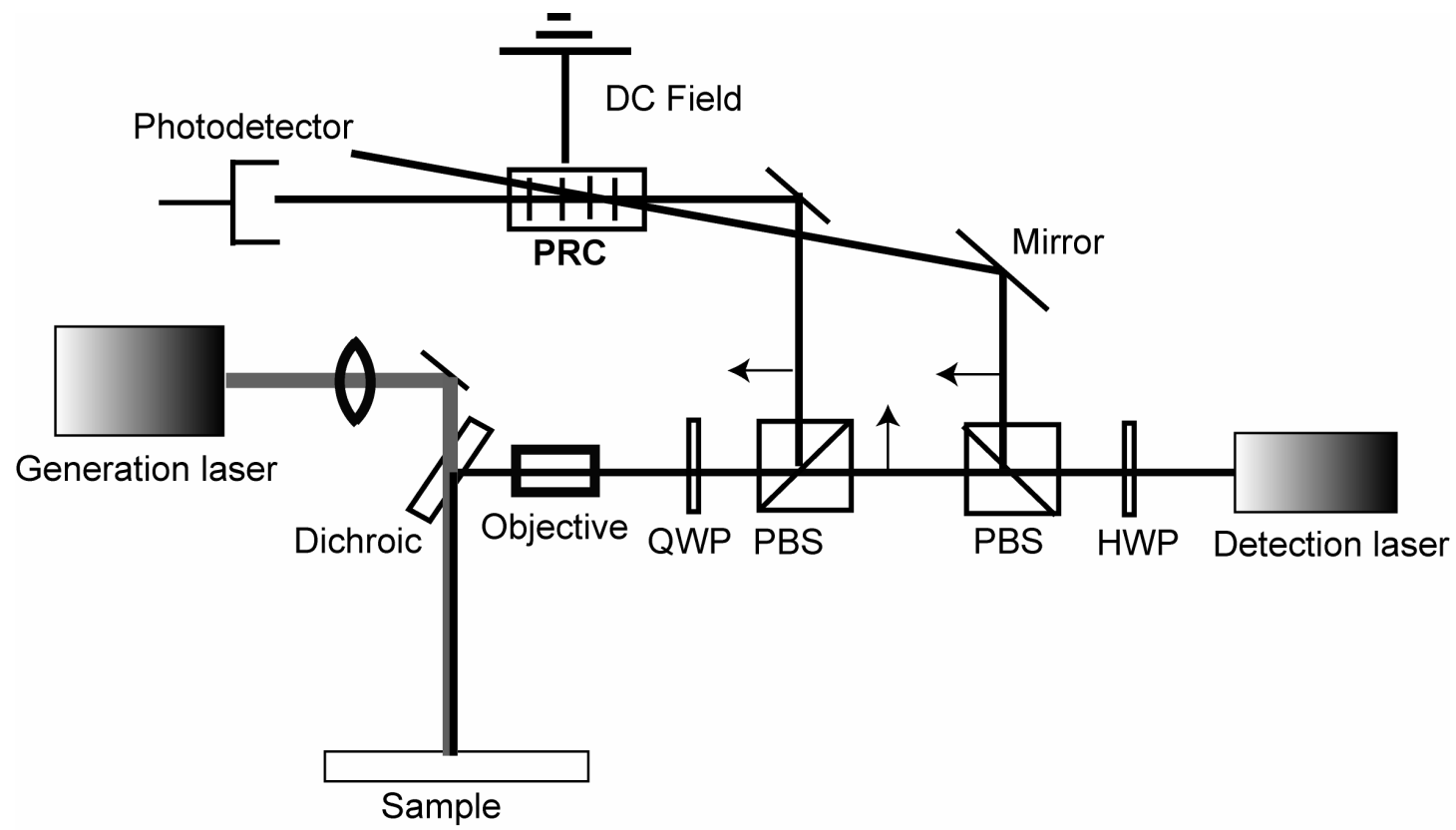

Figure 5. Experimental setup, HWP: Halfwave plate, PBS: Polarizing beam splitter, QWP: Quarterwave plate, PRC: Photorefractive crystal

\section{PRELIMINARY EXPERIMENTAL RESULTS}

Preliminary experimental results are presented from two layered samples. The samples were fabricated from thin polymer neutral density filters and optically transparent polystyrene layers. The layers were bonded using a thin layer of adhesive (superglue). The laser source and receiver were co-aligned on the surface of the specimens. In the first sample, 
a single neutral density filter (absorption coefficient of $\sim 13 \mathrm{~mm}^{-1}$ ) was attached to an optically transparent substrate. In the second configuration, a $125 \mu \mathrm{m}$ polystyrene layer was placed on top of two neutral density filters with absorption coefficients of approximately $13 \mathrm{~mm}^{-1}$ and $43 \mathrm{~mm}^{-1}$, respectively. In the case of the theoretical results, several assumptions were made. First, it was assumed that each layer had the same thermo-acoustic properties, and these were taken as the properties of polystyrene. Next, some variability in the thickness and absorption coefficient of the absorbing layers was found, and this was compensated for in the model when these parameters were chosen. The laser source radius was taken as $115 \mu \mathrm{m}$ for the calculations. The experimental displacement traces are shown in Figure $6 \mathrm{a}, \mathrm{b}$ for the single and multiple layer absorber, respectively. The calculated displacements are given in Figures $6 \mathrm{c}, \mathrm{d}$. The absorption coefficient for the single layer was taken as $15 \mathrm{~mm}^{-1}$ with a layer thickness of $75 \mu \mathrm{m}$, and in the second sample with two absorbing layers, the absorption coefficient of the top layer was $12 \mathrm{~mm}^{-1}$ with a thickness of $95 \mu \mathrm{m}$, and the bottom was $48 \mathrm{~mm}^{-1}$ with a thickness of $95 \mu \mathrm{m}$. These values were chosen based on the experimental measurements. The shape of the measured waveforms agrees reasonably well with theory. The disagreement at later times is most likely due to multiple acoustic reflections from the substrate and possibly between layers. The amplitude of the waveforms is considerably below that observed experimentally for both of the samples. This may be due in part to the fact that the thermal properties of the absorbing layer differ from those of polystyrene.
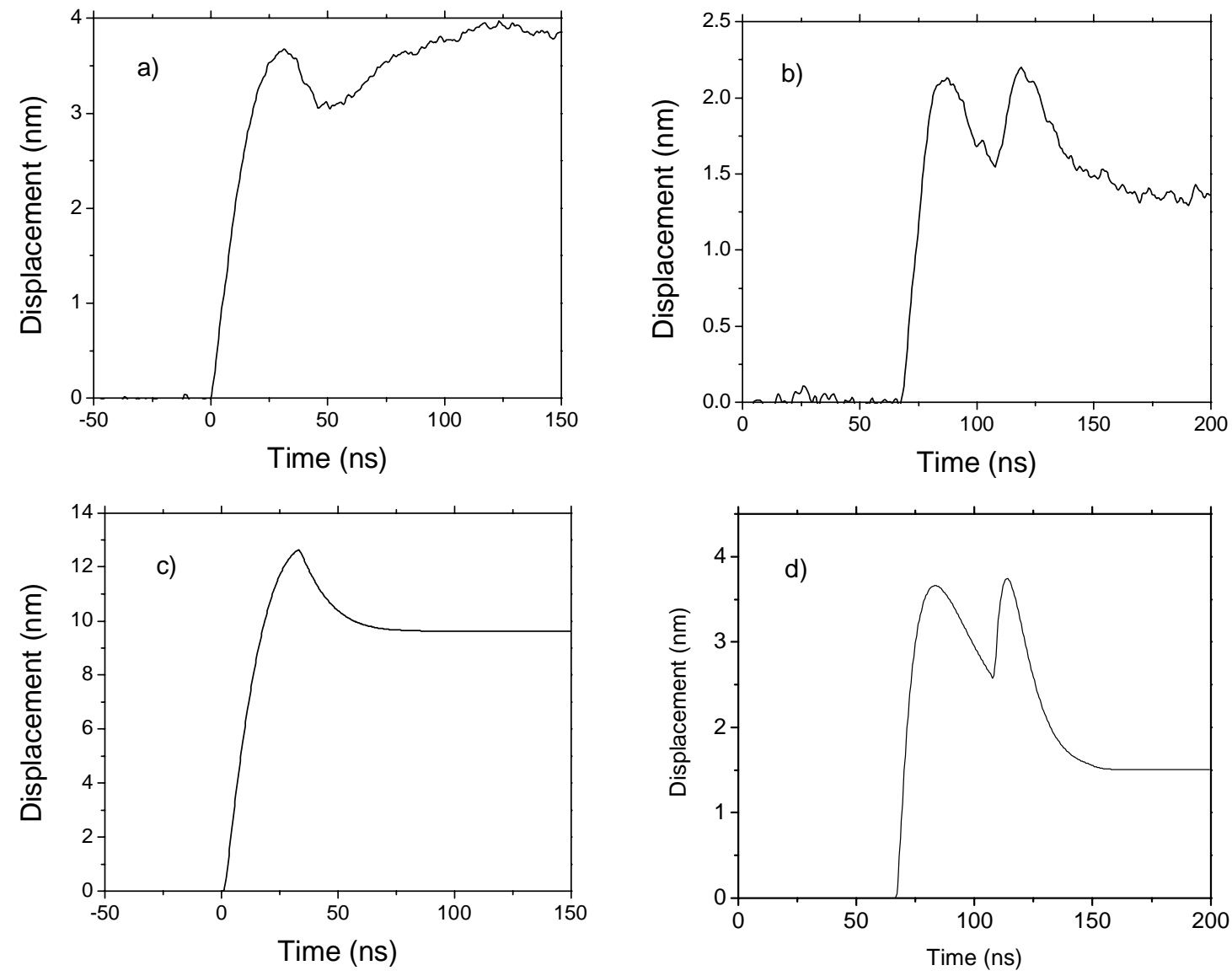

Figure 6. Comparison between experiment and theory for layered samples. a) and b) represent experimental waveforms on a homogeneously absorbing sample and a layered sample respectively. c) and d) represent the theoretical waveforms calculated for absorption distributions a) and b). 
The sensitivity of the system and the minimum detectable displacement is also analyzed for the present case. The signalto-noise ratio of the adaptive holographic interferometry system is given by [19]:

$$
S N R=\sqrt{\frac{2 \eta I_{d}(0,0) e^{-\alpha d}}{h v \Delta f}} \sin \left(\gamma^{\prime \prime} d\right) \frac{4 \pi \delta}{\lambda}
$$

where $\eta$ is the quantum efficiency of the photodetector, $I_{d}(0,0)$ is the intensity of the signal beam at the entrance of the crystal, $d$ is the thickness of the crystal through which light passes through and $\alpha$ the absorption coefficient of the crystal; hence $I_{d}(0,0) \mathrm{e}^{-\alpha d}$ is the light incident on the detector, $\gamma$ " is the imaginary part of the gain in the photorefractive crystal, $\Delta f$ is the bandwidth of the detector, $v$ is the optical frequency and $\lambda$ is the optical wavelength of the light. The SNR is directly proportional to the amount of light collected from the sample to the detector. Assuming that $\gamma$ ” $d=\pi / 2$, a quantum efficiency of 0.7 , a detection bandwidth of $200 \mathrm{MHz}$, the minimum detectable displacement (displacement for a SNR of 1) is calculated to be $0.0539 \mathrm{~nm}$.

\section{CONCLUSIONS}

A non-contact, non-invasive, high-resolution optical detection system based on photorefractive crystal based interferometry has been developed for optoacoustic microscopy. The system enables detection of surface displacements from a diffusely reflecting surface, and offers broadband $(200 \mathrm{MHz})$ detection. A 2-D theoretical model has been used to predict the out of plane displacement at the surface of target materials in various buried source geometries. The temporal and spatial profiles of the laser pulse are incorporated into the model. Polymer samples with a throughthickness absorption distribution were tested, and the agreement between theory and the experiment is reasonable. The preliminary results indicate that optical detection of optoacoustic transients is a promising technique for near surface imaging applications.

\section{ACKNOWLEDGEMENT}

This work was supported by CenSSIS, the Center for Subsurface Sensing and Imaging Systems, under the Engineering Research Centers Program of the National Science Foundation (award number EEC-9986821).

\section{REFERENCES}

1. A.A. Karabutov, E.V. Savateeva, N.B.Podymova., "Backward mode detection of laser-induced wide-band ultrasonic transients with optoacosutic transducer,”,J. Appl. Phys. 87(4), pp. 2003-2014, (2000).

2. C.G.A. Hoelen, F.F.M. de Mul., "Image reconstruction for photoacoustic scanning of tissue structures,", Appl Opt. 39(31), pp. 5872-5883, (2000).

3. G. Ku, X. Wang, G. Stoica, L. H. Wang., "Multiple-bandwidth photoacoustic tomography," Phys. Med. Biol. 49, 1329-1338, (2004).

4. J.D. Hamilton, G.L. Vossler M. O'Donnell., "High frequency ultrasound imaging using an active optical detector," IEEE Transactions on Ultrasonics, Ferroelectrics and Frequency Control, 45(3), pp. 719-727 (1998).

5. J.D. Hamilton, T. Buma, M. Spisar, M. O’Donnell., "High Frequency optoacoustic Arrays Using Etalon Detection," in IEEE Transactions on ultrasonics, ferroelectrics, and frequency control, 47 (1), pp. 160-169 (2000).

6. G. Paltauf, H. Schmidt-Kloiber, K.P. Kostli, K.P. M. Frenz., "Optical methods for two-dimenstional ultrasonic detecion," Appl. Phys. Lett. 75 (8), 1048-1050 (1999).

7. E. Z. Zhang, P. Beard., "Ultra high sensitivity, wideband Fabry Perot ultrasound sensors as an alternative to piezoelectric PVDF transducers for biomedical photoacoustic detection," in Proc. SPIE, 5320, pp. 222-229, (2001). 
8. PC. Beard., "Interrogation of free-space Fabry-Perot sensing interferometers by angle tuning," Meas. Sci. Technol. 14, pp. 1998-2005, (2003).

9. B.P. Payne, V. Venugopalan, B.B. Mikic, N.S. Nishioka., "Optoacoustic tomography using time-resolved interferometric detection of surface displacement," in J. Biomed. Opt. 8(2), 273-280 (2003).

10. S.A. Carp, A. Guerra, S.Q. Duque, V. Venugopalan., "Optoacoustic imaging using interferometric measurement of surface displacement," App. Phys. Lett. 85(23), 5772-5774 (2004).

11. V.E. Gusev, and A.A. Karabutov., Laser Optoacoustics, AIP Press, NewYork, (1993).

12. G.J. Diebold, T. Sun., "Properties of Photoacoustic Waves in One, Two, and Three Dimensions," Acustica 80, pp. 339-351, (1994).

13. K.L. Telschow, R.J. Conant, "Optical and thermal parameter effects on laser-generated ultrasound," J. Acoust. Soc. Am. 88(3), 1494-1502 (1990).

14. G.N. Maguluri, L.Sui, R.A. Roy, and T.W. Murray., "Optoacoustic systems for subsurface materials characterization," Review of Progress in QNDE, 23, eds. D.O Thomson and D.E. Chimenti, (2003).

15. J.B. Spicer Laser Ultrasonics In Finite Structures: Comprehensive Modelling with supporting Experiment. Ph.D Thesis, John Hopkins University (1991).

16. M. Oksanen and J.Wu, "Prediction of the temporal shape of an ultrasonic pulse in a photoacoustic sending application," Ultrasonics 32(1) pp.43-46, (1994).

17. A.A. Oraevsky, S.L. Jacques, and F.K., Tittel, "Measurement of tissue optical properties by time-resolved detection of laser-induced transient stress," Appl. Opt. 36(1), pp. 402-415, (1997).

18. M.A. Schweitzer, J.F. Power., "Optical depth profiling of thin films by impulse mirage effect spectroscopy. 1. Theory," Applied Spectroscopy 48(9), pp. 1054-1075, (1994).

19. P. Delaye, A. Blouin, D. Drolet, L. de Montmorillon, G. Roosen, J.-P.Monchalin., "Detection of ultrasonic motion of a scattering surface by photorefractive InP:Fe under an applied dc field," J. Opt. Soc. Am. B, 14(7), (1997). 